\title{
The shear-driven Rayleigh problem for generalised Newtonian fluids
}

\author{
Brian R. Duffy ${ }^{\mathrm{a}}$, David Pritchard ${ }^{\mathrm{a}, *}$, Stephen K. Wilson ${ }^{\mathrm{a}}$ \\ ${ }^{a}$ Department of Mathematics and Statistics, University of Strathclyde, 26 Richmond St, \\ Glasgow G1 1XH, Scotland, U. K.
}

\begin{abstract}
We consider a variant of the classical 'Rayleigh problem' ('Stokes's first problem') in which a semi-infinite region of initially quiescent fluid is mobilised by a shear stress applied suddenly to its boundary. We show that self-similar solutions for the fluid velocity are available for any generalised Newtonian fluid, regardless of its constitutive law. We demonstrate how these solutions may be used to provide insight into some generic questions about the behaviour of unsteady, non-Newtonian boundary layers, and in particular the effect of shear thinning or thickening on the thickness of a boundary layer.
\end{abstract}

Keywords: generalised Newtonian fluid, Rayleigh problem, Stokes's first problem, boundary layer

\section{Introduction: the Rayleigh problem}

The Rayleigh problem, sometimes called Stokes's first problem, was first formulated as a note to the celebrated paper by Stokes [1], and later discussed more fully by Rayleigh [2]. The problem is to describe the behaviour of a semi-infinite region of fluid ( $y<0$ in the notation we will use), bounded by a plane wall at $y=0$ and initially at rest, when the wall is impulsively accelerated to move in its own plane at a constant speed $U$.

For a Newtonian fluid, the momentum equation in the $x$-direction reduces

${ }^{*}$ Corresponding author. E-mail: david.pritchard@strath.ac.uk. Tel.: $(+44)(0) 141548$ 3819 . 
to the linear diffusion equation

$$
\frac{\partial u}{\partial t}=\nu \frac{\partial^{2} u}{\partial y^{2}}
$$

where the velocity $\mathbf{u}=u(y, t) \mathbf{i}$ and $\nu$ is the constant kinematic viscosity. The velocity field then has a self-similar form (see e.g. Drazin \& Riley [3, section $4.2]$ ),

$$
u(y, t)=U(1+\operatorname{erf}(\eta)), \quad \text { where } \quad \eta=\frac{y}{2(\nu t)^{1 / 2}},
$$

and where erf is the standard error function. Drazin \& Riley [3] also note that by a simple change of variables the solution (2) may be used to describe the case in which, instead of being driven by a velocity applied suddenly at $y=0$, the flow is driven by a shear stress of magnitude $\tau_{0}$ applied suddenly at $y=0$. The solution in this case is given by

$\frac{\partial u}{\partial y}=\frac{\tau_{0}}{\rho \nu}(1+\operatorname{erf}(\eta)) \quad$ and $\quad u(y, t)=\frac{2 \tau_{0}}{\rho \sqrt{\nu}} t^{1 / 2}\left[\eta(1+\operatorname{erf}(\eta))+\frac{1}{\sqrt{\pi}} \exp \left(-\eta^{2}\right)\right]$,

where $\rho$ is the fluid density and where $\eta$ is defined as above.

Aside from its value as an exact solution to the Navier-Stokes equations, equation (2) provides a useful paradigm for certain aspects of boundary-layer flow, and is often used pedagogically to illustrate the concepts of momentum and vorticity diffusion. The Rayleigh problem is thus a valuable starting point when we consider how non-Newtonian effects may modify the structure of unsteady boundary layers, and insight from this problem may complement that gained from studies of steady, spatially-developing boundary layers in non-Newtonian fluids [e.g. 4, 5]. It is important to note that the Rayleigh problem considers the particular case in which the 'outer' flow, far from the boundary, is zero, and that in more complicated scenarios matching the inner and outer flows for non-Newtonian fluids may be a non-trivial task [5].

A large number of variations on the Rayleigh problem have been investigated, and we will not attempt to provide a comprehensive review. We will concern ourselves here only with the generalisations from Newtonian to non-Newtonian fluids, and with those studies that have sought to develop exact or asymptotic solutions rather than fully numerical solutions.

In the earliest such study, Bird [6] demonstrated that for a power-law (Ostwald-de Waele) fluid, the equation corresponding to the velocity diffusion equation (1) is non-linear, but still admits self-similar solutions analogous to equation (2). Bird presented solutions for several shear-thinning 
cases, demonstrating that the more strongly shear-thinning the fluid is, the more gradually the velocity decays with distance from the boundary. Wein \& Mitschka [7] subsequently used this work to obtain approximate solutions to the Rayleigh problem for more general rheological models. Pascal $[8,9]$ re-derived the power-law solution, added a yield stress, and considered the behaviour of the solutions for shear-thickening fluids. For a shear-thickening power-law fluid the boundary layer is strictly finite; that is, at any instant the velocity is identically zero beyond a certain distance from the boundary, which emerges as part of the solution to the problem [8]. An analogous finitewidth boundary layer occurs at leading order in the solutions for a steady, spatially-developing boundary-layer flow in shear-thickening power-law fluids $[4,5]$.

The Rayleigh problem has also been extensively investigated for classes of viscoelastic fluids for which the momentum equation for unsteady rectilinear flow reduces to a linear PDE. The first study of this kind was by Tanner [10], who investigated the Rayleigh problem for an Oldroyd-B fluid. Among the extensive literature that has since developed, key studies have been those by Rajagopal [11] for a second-grade fluid and by Phan-Thien \& Chew [12] for a Phan-Thien-Tanner fluid. Christov [13] provides a discussion and critique of much of the more recent work on the viscoelastic Rayleigh problem. In addition, the Rayleigh problem has been extended to more complex fluids, including a model of a concentrated suspension [14] and of a nematic liquid crystal [15]; in the former case, self-similar solutions are again available.

From a physical point of view, the classical Rayleigh problem may not be the most natural one to specify, because in practice it is often easier to apply a controlled shear stress than a controlled velocity to the boundary of a fluid. Nevertheless, studies of the Rayleigh problem have confined themselves almost exclusively to the velocity-driven, rather than the shear-driven, version, perhaps because these problems are essentially equivalent in the Newtonian case. In the non-Newtonian case, however, these problems are no longer equivalent. In the present work, we will demonstrate that self-similar solutions to the shear-driven problem may be obtained for any generalised Newtonian rheology; in contrast, such solutions do not in general exist for the velocity-driven problem. Although we will illustrate the solution approach for Carreau and power-law fluids, we emphasise that it is equally applicable to any generalised Newtonian fluid. We will derive the form of the self-similar solutions in section 2, and demonstrate in section 3 how they may be used to explore some generic questions that may be asked concerning unsteady 
non-Newtonian boundary layers.

\section{Problem specification and governing equations}

2.1. Two-dimensional unsteady rectilinear flow of a generalised Newtonian fluid

The mass-conservation and momentum-balance equations for a fluid of constant density $\rho$, when body forces are neglected, are

$$
\nabla \cdot \mathbf{u}=0
$$

and

$$
\rho \frac{\mathrm{D} \mathbf{u}}{\mathrm{D} t}=-\nabla p+\nabla \cdot \boldsymbol{\sigma}
$$

where $\mathbf{u}, p$ and $\boldsymbol{\sigma}$ are the velocity, pressure and extra-stress tensor of the fluid, and $t$ denotes time.

A generalised Newtonian fluid is one for which the constitutive equation takes the form

$$
\boldsymbol{\sigma}=2 \mu(q) \mathbf{e}
$$

where $\mathbf{e}$ is the rate-of-strain tensor, given by

$$
\mathbf{e}=\frac{1}{2}\left(\nabla \mathbf{u}+(\nabla \mathbf{u})^{\mathrm{T}}\right)
$$

$q$ is the local shear rate, given by $q=\left(2 \operatorname{tr}\left(\mathbf{e}^{2}\right)\right)^{1 / 2}$, and $\mu=\mu(q)$ is a prescribed shear-rate-dependent viscosity function. The quantity $\tau=\left(\frac{1}{2} \operatorname{tr}\left(\boldsymbol{\sigma}^{2}\right)\right)^{1 / 2}$ $=\mu(q) q$ provides a measure of the local extra stress.

For two-dimensional unsteady rectilinear flow with velocity of the form $\mathbf{u}=u(y, t) \mathbf{i}$ referred to Cartesian coordinates $O x y z$ we have $\nabla \cdot \mathbf{u}=0$ and $\mathbf{u} \cdot \nabla \mathbf{u}=\mathbf{0}$ identically, and the only non-zero components of $\boldsymbol{\sigma}$ are

$$
\sigma_{12}=\sigma_{21}=\mu(q) \frac{\partial u}{\partial y}, \quad \text { where } \quad q=\left|\frac{\partial u}{\partial y}\right| .
$$

Note that in such rectilinear flows the vorticity $\omega=\omega \mathbf{k}$, where $\omega=-\partial u / \partial y$, so $q=|\boldsymbol{\omega}|$. For flows in which the pressure gradient $\partial p / \partial x$ is zero, equation (5) reduces to the nonlinear parabolic equation

$$
\rho \frac{\partial u}{\partial t}=\frac{\partial}{\partial y}\left(\mu(q) \frac{\partial u}{\partial y}\right)
$$

When the fluid is Newtonian, $\mu(q)=\rho \nu$, a constant, and (9) reduces immediately to (1). 


\subsection{The shear-driven Rayleigh problem}

We consider the situation in which fluid occupies the half space $y \leq 0$, with a boundary at $y=0$. Specifically, we consider the problem in which the fluid is stationary for $t<0$ but for $t \geq 0$ is caused to flow with velocity $\mathbf{u}=u(y, t) \mathbf{i}$ by a constant shear stress $\tau_{0}>0$ in the $x$ direction applied at the boundary $y=0$. Thus at $y=0$ we have the boundary condition

$$
\sigma_{12}=\sigma_{21}=\left\{\begin{aligned}
0 & \text { if } t<0, \\
\tau_{0} & \text { if } t \geq 0,
\end{aligned} \quad \text { or, equivalently, } \quad \frac{\partial u}{\partial y}=\left\{\begin{aligned}
0 & \text { if } t<0 \\
q_{0} & \text { if } t \geq 0
\end{aligned}\right.\right.
$$

where the positive constants $\tau_{0}$ and $q_{0}$ are related by

$$
\tau_{0}=\mu\left(q_{0}\right) q_{0}
$$

We seek solutions for which $\partial u / \partial y \geq 0$ everywhere and so we may take $q=\partial u / \partial y \geq 0$; the velocity $u$ must then be maximum at $y=0$, and we may reasonably expect the shear rate $q$ also to be highest at the boundary, although we do not require this.

\subsubsection{Nondimensionalisation}

We nondimensionalise variables via

$$
y=L y^{*}, \quad t=\frac{\rho L^{2}}{\mu_{\mathrm{r}}} t^{*}, \quad u=\frac{L \tau_{0}}{\mu_{\mathrm{r}}} u^{*}, \quad q=\frac{\tau_{0}}{\mu_{\mathrm{r}}} q^{*}, \quad \tau=\tau_{0} \tau^{*}, \quad \mu=\mu_{\mathrm{r}} \mu^{*},
$$

where $\mu_{\mathrm{r}}$ is an appropriate 'reference' viscosity (for example, the zero-shearrate viscosity) and $L$ is an arbitrary lengthscale. Note that the dimensionless shear stress $\tau^{*}$ is not an additional variable but is given by $\tau^{*}\left(q^{*}\right)=\mu^{*}\left(q^{*}\right) q^{*}$.

The non-dimensionalisation (12) contains the artificial lengthscale $L$, which remains undetermined by the boundary and initial conditions. This is an indication that self-similar solutions can be found. The only combination of $y^{*}$ and $t^{*}$ that is independent of $L$ is $y^{*} / t^{* 1 / 2}=\left(\rho / \mu_{\mathrm{r}}\right)^{1 / 2} y / t^{1 / 2}$, while the only combination of $u^{*}$ and $t^{*}$ that is independent of $L$ is $u^{*} / t^{* 1 / 2}=$ $\left(\left(\rho \mu_{\mathrm{r}}\right)^{1 / 2} / \tau_{0}\right) u / t^{1 / 2}$. Requiring that when a solution $u(y, t)$ is non-dimensionalised the corresponding solution $u^{*}\left(y^{*}, t^{*}\right)$ should not depend on $L$ thus leads us to consider solutions of the self-similar form

$$
u^{*}=2 t^{* 1 / 2} f(\eta), \quad \text { where } \quad \eta=\frac{y^{*}}{2 t^{* 1 / 2}},
$$


and where the factors of 2 have been introduced for convenience. For solutions of this form, $q^{*}=f^{\prime}(\eta)$ and $\mu^{*}=\mu^{*}\left(f^{\prime}\right)$ are also independent of $L$.

Although $\mu_{\mathrm{r}}$ is not an artificial scale in the sense that $L$ is, it is not uniquely defined. In particular, if we wish to compare results for different constitutive laws $\mu(q)$, this will naturally lead us to compare solutions for which $\mu_{\mathrm{r}}$ is defined in different ways. There is then a danger that the effects of changing the choice of $\mu_{\mathrm{r}}$ will be confused with the qualitative effects of nonNewtonian behaviour. To avoid this, in section 3.2 we will seek quantities that are independent both of $L$ and of $\mu_{\mathrm{r}}$. In particular, the only combination of $q^{*}$ and $\eta$ that is independent of $\mu_{\mathrm{r}}$ is given by $\eta^{2} q^{*}=\left(\rho /\left(4 \tau_{0}\right)\right) y^{2} q / t$. A particular example of this combination of variables will be of interest when we examine boundary-layer thickness in section 3.2.

\subsubsection{The boundary-value problem for the self-similar solution}

With the non-dimensionalisation (12), equation (9) becomes

$$
\frac{\partial u^{*}}{\partial t^{*}}=\frac{\partial}{\partial y^{*}}\left(\mu^{*}\left(q^{*}\right) \frac{\partial u^{*}}{\partial y^{*}}\right), \quad \text { where } \quad q^{*}=\frac{\partial u^{*}}{\partial y^{*}} .
$$

Seeking a solution of the form (13) to equation (14), we find that the unknown function $f(\eta)$ must satisfy the nonlinear ordinary differential equation

$$
2\left(f-\eta f^{\prime}\right)=\left[\mu^{*}\left(f^{\prime}\right) f^{\prime}\right]^{\prime} .
$$

Equation (15) is to be integrated subject to the boundary conditions

$$
f^{\prime}(0)=q_{0}^{*}, \quad \text { and } \quad f(\eta) \rightarrow 0 \quad \text { as } \quad \eta \rightarrow-\infty,
$$

where $q_{0}^{*}=q_{0} \mu_{\mathrm{r}} / \tau_{0}$, and $q_{0}$ is defined by (11), so in dimensionless terms

$$
\mu^{*}\left(q_{0}^{*}\right) q_{0}^{*}=1 .
$$

Numerical solutions to the boundary-value problem (15)-(17) may be obtained, once $\mu^{*}\left(q^{*}\right)$ is specified, using standard software packages such as Maple or Mathematica; we present several examples in the following section, but we emphasise that these are not the only rheologies for which solutions can be obtained.

Note that such self-similar solutions can be constructed for general $\mu^{*}\left(q^{*}\right)$ only when the flow is forced by a suddenly applied shear stress (or, equivalently, a suddenly applied shear rate) at $y=0$. In particular, it is not 
generally possible to construct such solutions for the classical version of the Rayleigh problem in which the velocity at $y=0$ is suddenly increased. In solutions to this classical problem, $q^{*}$ is necessarily a function of $t^{*}$ as well as of $\eta$; now, $t^{*}$ can be eliminated from the governing equation (14) only if $\mu^{*}\left(q^{*}\right)$ can be written as the product of a function of $\eta$ and a single power of $t^{*}$, and this is possible only when $\mu^{*}\left(q^{*}\right)$ is a monomial in $q^{*}$ (as in [8]).

It is also of interest that if equation (14) is differentiated with respect to $y^{*}$ then it can be written as a concentration-dependent diffusion equation for $q^{*}$ :

$$
\frac{\partial q^{*}}{\partial t^{*}}=\frac{\partial}{\partial y^{*}}\left(D\left(q^{*}\right) \frac{\partial q^{*}}{\partial y^{*}}\right), \quad \text { where the diffusivity } \quad D\left(q^{*}\right)=\frac{\mathrm{d} \tau^{*}}{\mathrm{~d} q^{*}} .
$$

The problem we consider here is thus formally identical to some of those investigated by Philip [16] (see also Witelski [17]); our approach differs from theirs, however, in that we explore the properties of the solution on the understanding that $\mu^{*}\left(q^{*}\right)$ is specified, rather than by seeking forms of $D^{*}\left(q^{*}\right)$ that yield exact solutions for $q^{*}$. We also note that a mathematically equivalent problem arises in simple models of turbulent shear flow, in which self-similar solutions are again available only for shear-forced problems [18].

Equation (18) is also informative because it provides a natural way to write the problem for rheological models that specify the shear rate as a function of the shear stress, $q=q(\tau)$. For such models, (18) may be written as

$$
q^{* \prime}\left(\tau^{*}\right) \frac{\partial \tau^{*}}{\partial t^{*}}=\frac{\partial^{2} \tau^{*}}{\partial y^{* 2}}
$$

and similarity solutions may be developed as above. Furthermore, equation (18) indicates, perhaps surprisingly, that the presence of a yield stress does not fundamentally change the nature of the solutions. For a yield-stress fluid (such as the Herschel-Bulkley fluid) with a constitutive law that reduces in simple shear to $\tau=\tau_{\mathrm{y}}+\mu(q) q$, it is clear from (18) that $D\left(q^{*}\right)$ is determined only by $\mu^{*}\left(q^{*}\right)$ and the yield stress is irrelevant. The solution for a HerschelBulkley fluid is therefore equivalent to that for a power-law fluid (and the solution for a Bingham fluid is equivalent to that for a Newtonian fluid) subject to the boundary condition $\tau=\tau_{0}-\tau_{\mathrm{y}}$ for $t>0$, provided only that $\tau_{0}>\tau_{\mathrm{y}}$. This equivalence was noted by Pascal $[8,9]$, and so we do not pursue the point further. 


\section{Specific solutions and their properties}

\subsection{Low-shear behaviour and finite boundary layers}

Pascal [8] presented explicit solutions to the velocity-driven Rayleigh problem for power-law fluids, in which $\mu(q)=\mu_{n} q^{n-1}$, where $n$ is the powerlaw index and $\mu_{n}$ is a dimensional consistency parameter. In this case, equation (14) becomes a form of the 'porous medium equation', and an interesting feature of the solutions is that for shear-thickening fluids, for which $n>1$, the boundary layer is finite. (The behaviour of compactly-supported solutions to the porous medium equation has been extensively studied: see for example [19].) A finite boundary layer is also found under oscillatory forcing (Stokes's second problem) for a shear-thickening power-law fluid [20] and in steady boundary-layer flow of a shear-thickening power-law fluid $[4,5]$.

In the light of these results for power-law fluids, it is natural to ask what properties of a general viscosity function $\mu^{*}\left(q^{*}\right)$ are required in order for finite boundary layers to exist, and the boundary-value problem (15)-(17) provides a useful way to approach this question. We assume that at small shear rates, $q^{*} \rightarrow 0$, the asymptotic behaviour of the viscosity is $\mu^{*}\left(q^{*}\right) \sim A q^{* \alpha}$ for some positive constant $A$; although $\alpha$ may be positive, negative or zero, it is physically realistic to require that $\alpha>-1$ so that $\tau^{*}$ is an increasing function of $q^{*}$ for small $q^{*}$. We will in turn postulate a finite boundary layer and a far-field decay of the velocity, and we will obtain the conditions on $\alpha$ that are required for each type of behaviour to occur.

(i) A finite boundary layer. We postulate a finite boundary layer solution of the form

$$
f(\eta) \sim B\left(\eta-\eta_{0}\right)^{k} \quad \text { as } \quad \eta \rightarrow \eta_{0}^{+},
$$

where the edge of the boundary layer is at $\eta=\eta_{0}<0$, and where $B$ and $k$ are positive constants. For simplicity, we write $\epsilon=\eta-\eta_{0}>0$. Expanding (15) in powers of $\epsilon$ and retaining only the leading-order terms, we find that

$$
-2 \eta_{0} a k \epsilon^{k-1} \sim A(B k)^{\alpha+1}(\alpha+1)(k-1) \epsilon^{(\alpha+1)(k-1)-1} .
$$

Equating the powers of $\epsilon$, we deduce that $k=(\alpha+1) / \alpha$, and the requirement that $k>0$ then yields the condition $\alpha>0$. The constants $B$ and $\eta_{0}$ are therefore related by

$$
-2 \eta_{0}=A B^{\alpha}\left(\frac{\alpha+1}{\alpha}\right)^{\alpha+1},
$$


and we note that $\eta_{0}<0$ as required. We also note that for a power-law fluid $\alpha=n-1$, so the condition $\alpha>0$ yields $n>1$ (shear thickening) consistently with the results of [8].

(ii) Algebraic decay in the far field. We now postulate a far-field solution of the form

$$
f(\eta) \sim-\frac{C}{\gamma+1}(-\eta)^{\gamma+1}, \quad \text { so that } \quad f^{\prime}(\eta) \sim C(-\eta)^{\gamma}, \quad \text { as } \quad \eta \rightarrow-\infty,
$$

where $\gamma<0$ and $C>0$ are constants. Expanding (15) in powers of $-\eta$ and retaining leading-order terms, we find that

$$
\frac{2 C \gamma}{\gamma+1}(-\eta)^{\gamma+1} \sim-A C^{\alpha+1} \gamma(\alpha+1)(-\eta)^{\alpha \gamma+\gamma-1}
$$

Equating the powers of $-\eta$, we deduce that $\gamma=2 / \alpha$, and the requirement that $\gamma<0$ then yields the condition $\alpha<0$. The constant $C$ is then given by

$$
\frac{2 \alpha}{(\alpha+2)(\alpha+1)}=-A C^{\alpha}
$$

and we note that $C>0$ as long as $-1<\alpha<0$. We also note that for a power-law fluid, the condition $\alpha<0$ yields $n<1$ (shear thinning), again consistently with the results of [8].

(iii) Newtonian behaviour. If the viscosity tends to a constant at small shear rates, i.e. in the special case $\alpha=0$, then Newtonian behaviour is recovered and the velocity decays as $\exp \left(-\eta^{2}\right) / \eta^{2}$ at large distances from the boundary. Note that this Newtonian limiting behaviour is consistent with our results as $\alpha \rightarrow 0$ in both the shear-thinning and shear-thickening regimes. In the shear-thickening regime, equation (22) reduces to $\eta_{0} \sim-\frac{1}{2} A((\alpha+$ $1) / \alpha)^{\alpha+1}$, so $\eta_{0} \rightarrow-\infty$ as $\alpha \rightarrow 0^{+}$. Consequently, as the shear-thickening solutions approach the Newtonian limit, the limit of the finite boundary layer diverges to infinity, consistently with the existence of an 'infinitely wide' boundary layer in the Newtonian case. Conversely, in the shear-thinning regime, the decay rate at large values of $|\eta|$ is given by $\gamma=2 / \alpha$. As $\alpha \rightarrow 0^{-}$, $\gamma \rightarrow-\infty$, so the algebraic decay becomes faster and faster, until when $\alpha=0$ the algebraic decay has entirely collapsed; this is consistent with the superexponential decay in the Newtonian case $\alpha=0$.

Finally, we note that although other low-shear-rate behaviours for $\mu^{*}\left(q^{*}\right)$ could be postulated, the three regimes defined by $-1<\alpha<0, \alpha=0$ and $\alpha>0$ cover the vast majority of commonly employed rheological models. 


\subsection{Shear thinning or thickening and boundary-layer thickness}

With the exception of the power-law and Herschel-Bulkley fluids, most rheological models possess a non-zero limiting viscosity at low shear rates, $\mu(q) \rightarrow \mu_{0}$ as $q \rightarrow 0$. Consequently, at sufficient distances from the boundary such fluids will behave like Newtonian fluids with viscosity $\mu_{0}$, but closer to the boundary non-Newtonian effects will become apparent. The self-similar solutions described in section 2.1 allow us readily to quantify this distance and thus to determine the importance of the non-Newtonian behaviour. In particular, we address the following question: if the viscosity at high shear rates is lower (or greater) than that at low shear rates, how much narrower (or wider) is the boundary layer in the Rayleigh problem than an estimate based on the limiting low-shear viscosity would suggest?

\subsubsection{The displacement thickness}

To answer this question we require a robust definition of the boundarylayer thickness. A natural definition to choose is the velocity displacement thickness

$$
\delta(t)=\frac{1}{u(0, t)} \int_{-\infty}^{0} u(y, t) \mathrm{d} y,
$$

which for self-similar solutions of the form (13) can be written as

$$
\delta(t)=2 \sqrt{\frac{\mu_{\mathrm{r}}}{\rho}} t^{1 / 2} \eta_{\mathrm{d}}, \quad \text { where } \quad \eta_{\mathrm{d}}=\frac{1}{f(0)} \int_{-\infty}^{0} f(\eta) \mathrm{d} \eta .
$$

The displacement thickness $\delta(t)$ is precisely analogous to the familiar velocity displacement thickness in classical boundary-layer theory [e.g. 21, section 5.8], while the dimensionless coefficient $\eta_{\mathrm{d}}$ defines a value of $\eta$ that characterises the thickness of the boundary layer.

For a Newtonian fluid with constant viscosity $\mu_{\mathrm{r}}$, the boundary-value problem (15)-(17) has the solution

$$
f(\eta)=\eta(1+\operatorname{erf}(\eta))+\frac{1}{\sqrt{\pi}} \exp \left(-\eta^{2}\right)
$$

(cf. (3)), for which

$$
\eta_{\mathrm{d}}=\frac{\sqrt{\pi}}{4} \approx 0.443
$$

Note that the result (29) depends on the viscosity $\mu_{\mathrm{r}}$ employed in the nondimensionalisation. However, recalling the discussion from section 2.2.1, we 
conclude that quantities of the form $\eta_{\mathrm{d}}^{2} q^{*}$ are independent of the choice of $\mu_{\mathrm{r}}$. In particular, the product of $\eta_{\mathrm{d}}^{2}$ with the shear rate $q_{0}^{*}$ at the surface,

$$
\mathcal{F}=\eta_{\mathrm{d}}^{2} q_{0}^{*}=\eta_{\mathrm{d}}^{2} f^{\prime}(0),
$$

is independent of $\mu_{\mathrm{r}}$, and takes the value $\mathcal{F}=\pi / 16$ for any Newtonian fluid. In the investigation of non-Newtonian fluids below, we will seek to separate the effects of non-Newtonian behaviour from those of the choice of $\mu_{\mathrm{r}}$. The quantity $\mathcal{F}$ defined by (30) will be useful in doing so, because any deviation of $\mathcal{F}$ from the value $\mathcal{F}=\pi / 16$ must be due to non-Newtonian effects.

\subsubsection{Results for a Carreau fluid}

To illustrate the effect of shear thinning and thickening on the displacement thickness, we consider a specific rheology, the Carreau model [e.g. 22, section 1.5]. In dimensional form the Carreau model may be written as

$$
\mu(q)=\mu_{\infty}+\frac{\mu_{0}-\mu_{\infty}}{\left[1+(\lambda q)^{2}\right]^{(1-n) / 2}}
$$

where $n<1$, where $\mu_{0}$ and $\mu_{\infty}$ are the limiting viscosities at low and high shear rates respectively, and where the inverse reference shear rate $\lambda$ has the dimension of time. We take the reference viscosity for non-dimensionalisation to be $\mu_{\mathrm{r}}=\mu_{0}$, so the dimensionless viscosity becomes

$$
\mu^{*}\left(q^{*}\right)=\mu_{\infty}^{*}+\frac{1-\mu_{\infty}^{*}}{\left[1+\left(\lambda^{*} q^{*}\right)^{2}\right]^{(1-n) / 2}},
$$

where we define

$$
\mu_{\infty}^{*}=\frac{\mu_{\infty}}{\mu_{0}} \quad \text { and } \quad \lambda^{*}=\frac{\lambda \tau_{0}}{\mu_{0}} .
$$

The extent to which non-Newtonian effects are evident depends on the magnitude of $\lambda^{*} q_{0}^{*}$. Recall that the shear rate is greatest at the boundary, $q^{*}=q_{0}^{*}$ at $y^{*}=0$. Thus if $\lambda^{*} q_{0}^{*} \ll 1$ then the term $\lambda^{*} q^{*}$ is negligible everywhere, and the fluid behaves simply like a Newtonian fluid with constant dimensionless viscosity 1 . If, conversely, $\lambda^{*} q_{0}^{*} \gg 1$, then close to the boundary we have $\mu^{*}\left(q^{*}\right) \sim \mu_{\infty}^{*}$; the initial decay of the velocity with distance from the boundary is therefore that of a Newtonian fluid with dimensionless viscosity $\mu_{\infty}^{*}$. This region of Newtonian behaviour extends until $\lambda^{*} q^{*} \sim 1$, when nonNewtonian effects assert themselves; there is then a region of non-Newtonian 
behaviour, and further still from the boundary the velocity must again follow the Newtonian solution with constant dimensionless viscosity 1.

Figures $1 \mathrm{a}-\mathrm{c}$ illustrate the profiles of velocity, shear rate (vorticity) and shear stress for a Newtonian fluid compared with two Carreau fluids, one strongly shear-thinning (in which $\lambda^{*} q_{0}^{*} \approx 42$ ) and one shear-thickening (in which $\lambda^{*} q_{0}^{*} \approx 2.3$ ). Shear thinning is associated with higher shear rates, correspondingly lower viscosities and consequently lower momentum diffusivity, so the disturbance is confined within a relatively thin boundary layer close to $y=0$. Shear thickening, conversely, is associated with lower shear rates, correspondingly higher viscosities and consequently higher momentum diffusivity, so the boundary layer is somewhat wider. The difference in boundary-layer thickness is most obvious in the plots of shear stress (figure $1 \mathrm{c})$, where it is not obscured by differences in the values of $f$ and $f^{\prime}$ at $\eta=0$. Figure $1 \mathrm{~d}$ illustrates the behaviour of the solutions close to the wall, demonstrating that as $\lambda$ increases the solutions for a Carreau fluid converge to that for the corresponding Newtonian fluid with $\mu^{*}=\mu_{\infty}^{*}$. As expected, this convergence is strongest close to the wall where $q^{*}$ is largest.

Figure 2 a shows how the displacement-thickness coefficient $\eta_{\mathrm{d}}$ varies with the rheological parameters $\mu_{\infty}^{*}$ and $\lambda^{*}$. Unsurprisingly, when either $\mu_{\infty}^{*}$ is close to unity (weak shear thinning or thickening) or $q^{*}$ is small (a weak applied shear stress), the non-Newtonian effects are negligible and $\eta_{\mathrm{d}}$ remains close to its Newtonian value $\eta_{\mathrm{d}} \approx 0.443$. Even outwith this regime, the variation of $\eta_{\mathrm{d}}$ is not strong: even when $\mu_{\infty}^{*}=0.1$ and $\lambda^{*}=10, \eta_{\mathrm{d}}$ remains more than half of its Newtonian value (cf. figure 1).

The variation of the shear rate at the boundary with the rheological parameters is closely correlated with that of $\eta_{\mathrm{d}}$. In fact, throughout the region of parameter space plotted, the relationship $\mathcal{F}=\pi / 16$, which would be exact for a Newtonian fluid, is approximately satisfied (figure $2 \mathrm{~b}$ ), with deviations everywhere smaller than $25 \%$. It is interesting that this result holds approximately even when the fluid is strongly non-Newtonian, and it offers a quick means of estimating the boundary-layer thickness in a Carreau fluid simply from the boundary condition on $q$, without having to solve the full boundary-value problem. Written in dimensional terms using (27) this result corresponds to the statement that

$$
\delta(t)=2 \sqrt{\frac{\mu_{0}}{\rho}} t^{1 / 2} \eta_{\mathrm{d}} \approx \frac{\sqrt{\pi \tau_{0}}}{2 \sqrt{\rho q_{0}}} t^{1 / 2}, \quad \text { where } \quad \mu\left(q_{0}\right) q_{0}=\tau_{0} .
$$




\subsubsection{Power-law fluids revisited}

The results presented above for Carreau fluids appear at first to contradict the finding (see [6], and $\S 3.1$ above) that for power-law fluids, increased shear thinning leads to more gradual decay in the far field, and thus to wider boundary layers. To quantify the latter point, figure 3 presents the variation of $\eta_{\mathrm{d}}$ with $n$ for shear-thinning power-law fluids with $\mu^{*}\left(q^{*}\right)=q^{* n-1}$; the corresponding results for shear-thickening cases require more effort to compute numerically because of the finite boundary layer, and are omitted here.

Figure 3 a shows how $\eta_{\mathrm{d}}$ varies with the power-law index $n$. This figure corresponds to figure 2 a for the Carreau fluid, but appears to show the opposite trend: the more strongly shear thinning the fluid is (the further to the left in each plot), the larger $\eta_{\mathrm{d}}$ becomes for a power-law fluid (figure 3 a), but the smaller it becomes for a Carreau fluid (figure 2 a). To resolve this seeming contradiction we must carefully consider the reference quantities chosen to non-dimensionalise the problem. The argument presented in the discussion of figure 1 above is premised on a non-dimensionalisation in which the limiting viscosity in the far field is the same for all fluids, so shear thinning or thickening corresponds to a decrease or an increase in the viscosity near to the wall. In contrast, for power-law fluids no equivalent non-dimensionalisation is possible: instead, the definition $\mu^{*}\left(q^{*}\right)=q^{* n-1}$ implicitly takes the viscosity at the wall for its reference value, fixing $\mu_{\mathrm{r}}=\mu_{n}^{1 / n} \tau_{0}^{1-1 / n}$ and $f^{\prime}(0)=q_{0}^{*}=1$ in the process. Thus, in the dimensionless problem, shear thinning corresponds to an increase in viscosity in the far field rather than to a decrease in viscosity close to the wall.

A more relevant comparison is between figures $3 \mathrm{~b}$ and $2 \mathrm{~b}$. In figure $3 \mathrm{~b}$, the quantity $\mathcal{F}$ defined by (30), which is independent of the choice of $\mu_{\mathrm{r}}$, is plotted as a function of $n$. The trend of $\mathcal{F}$ with increased shear thinning (lower $n$ ) in figure $3 \mathrm{~b}$ is the same as the trend of $\mathcal{F}$ with increased shear thinning (lower $\mu_{\infty}^{*}$ ) in figure $2 \mathrm{~b}$. This resolution of the seeming contradiction illustrates the important point that, because the parameters appearing in different rheological models have different roles, and even different dimensions, these models may be incommensurable unless the ways in which they are compared are carefully defined. 


\section{Summary}

The class of generalised Newtonian fluids is a large one, and it is not easy to make general statements about their flow, whether steady or unsteady. It is therefore noteworthy that for a variation of the classical Rayleigh problem, in which a deep fluid layer flows under the influence of a shear stress applied suddenly at the boundary, self-similar solutions are available for any generalised Newtonian fluid regardless of its constitutive law. In these solutions, both the velocity at the boundary and the thickness of the boundary layer always increase as the square root of time. Full numerical solutions for any given constitutive law may be obtained by solving the boundary-value problem defined by the second-order ODE (15) and the boundary conditions (16)-(17).

These self-similar solutions may provide a useful benchmark for simulations of generalised Newtonian fluids. They also provide a simple prototype for unsteady boundary-layer flow, and may be interrogated to yield generic insight into such flow. In particular, we have demonstrated that a finite boundary layer can be expected to occur in this problem for any rheology that shares the asymptotic behaviour of shear-thickening power-law fluids in which the viscosity decays to zero algebraically at low shear rates. We have also used the self-similar solutions to quantify the extent to which shearthinning and -thickening properties reduce or increase the boundary-layer thickness. In particular, for a Carreau fluid a prediction of boundary-layer thickness based on a result for a Newtonian fluid is shown to provide a reasonably accurate estimate even in strongly non-Newtonian regimes.

Our results for the boundary-layer thickness also illustrate the more general point that a question such as 'does shear thinning increase or decrease boundary-layer thickness?' may make sense only in the context of a particular rheological model rather than generically. In particular, results for a power-law fluid may be misleading because different degrees of shear thinning and thickening are represented in this model by changing $n$, and with it the dimension of the consistency $\mu_{n}$ and of any quantities that depend on $\mu_{n}$; this contrasts with more realistic models such as the Carreau fluid in which different degrees of shear thinning or thickening are represented by altering the magnitudes, but not the dimensions, of $\mu_{0}$ and $\mu_{\infty}$.

These solutions represent, of course, only a first step towards a full understanding of unsteady boundary layers in non-Newtonian fluids. Existing work on the steady boundary-layer flow of a power-law fluid [5] suggests that 
matching an unsteady boundary layer to a non-trivial outer flow may be a challenging task. A further aspect that deserves attention is the stability of generalised Newtonian boundary layers to perturbations: it is known that the basic solution in the Newtonian version of the Rayleigh problem can become susceptible to a Tollmien-Schlichting instability [23], and it seems plausible that analogous instabilities could occur in the generalised Newtonian problem as well.

Acknowledgements. This work was started while S. K. W. was a Visiting Fellow at the Isaac Newton Institute for Mathematical Sciences in Cambridge, England, U. K., as part of the programme on "Mathematical Modelling and Analysis of Complex Fluids and Active Media in Evolving Domains", and completed while he was a Leverhulme Trust Research Fellow (2013-2015) supported by award RF-2013-355, "Small Particles, Big Problems: Understanding the Complex Behaviour of Nanofluids".

\section{References}

[1] G. G. Stokes, On the effect of the internal friction of fluids on the motion of pendulums, Transactions of the Cambridge Philosophical Society 9 (1851) 8-106.

[2] Lord Rayleigh, On the motion of solid bodies through viscous liquid, Philosophical Magazine 21 (1911) 697-711.

[3] P. G. Drazin, N. Riley, The Navier-Stokes Equations: a classification of flows and exact solutions, number 334 in London Mathematical Society Lecture Note Series, Cambridge University Press, 2006.

[4] A. Acrivos, M. J. Shah, E. E. Petersen, Momentum and heat transfer in laminar boundary-layer flows of non-Newtonian fluids past external surfaces, AIChE Journal 6 (1960) 312-317.

[5] J. P. Denier, P. P. Dabrowski, On the boundary-layer equations for power-law fluids, Proceedings of the Royal Society of London A 460 (2004) 3143-3158.

[6] R. B. Bird, Unsteady pseudoplastic flow near a moving wall, AIChE Journal 5 (1959) 565-566. 
[7] O. Wein, P. Mitschka, Pseudoähnlichkeitslösung des Rayleighschen Problems für reinviskose nicht-newtonsche Flüssigkeiten, Rheologica Acta 17 (1978) 463-470. (In German. English title: "Pseudo-similarity solutions to the Rayleigh problem for non-Newtonian fluids".).

[8] H. Pascal, Propagation of disturbances in a non-Newtonian fluid, Physica D 39 (1989) 262-266.

[9] H. Pascal, Similarity solutions to some unsteady flows of non-Newtonian fluids of power law behaviour, International Journal of Non-Linear Mechanics 27 (1992) 759-771.

[10] R. I. Tanner, Note on the Rayleigh problem for a visco-elastic fluid, Zeitschrift für angewandte Mathematik und Physik XIII (1962) 573580 .

[11] K. R. Rajagopal, A note on unsteady unidirectional flows of a nonNewtonian fluid, International Journal of Non-Linear Mechanics 17 (1982) 369-373.

[12] N. Phan-Thien, Y. T. Chew, On the Rayleigh problem for a viscoelastic fluid, Journal of Non-Newtonian Fluid Mechanics 28 (1988) 117-127.

[13] I. C. Christov, Stokes' first problem for some non-Newtonian fluids: results and mistakes, Mechanics Research Communications 37 (2010) $717-723$.

[14] N. Phan-Thien, A. L. Graham, Rayleigh similarity solutions and boundary layer flow for concentrated suspensions, Journal of Non-Newtonian Fluid Mechanics 64 (1996) 157-171.

[15] M. Díez, C. Atkinson, The Rayleigh problem for nematic liquid crystals. Similarity solutions, International Journal of Engineering Science 39 (2001) 245-284.

[16] J. R. Philip, General method of exact solution of the concentrationdependent diffusion equation, Australian Journal of Physics 13 (1960) $1-12$.

[17] T. P. Witelski, Stopping and merging problems for the porous media equation, IMA Journal of Applied Mathematics 54 (1995) 227-243. 
[18] S. Crow, Turbulent Rayleigh shear flow, Journal of Fluid Mechanics 32 (1968) 113-130.

[19] K.-A. Lee, J. L. Vázquez, Geometrical properties of solutions of the porous medium equation for large times, Indiana University Mathematics Journal 52 (2003) 991-1016.

[20] D. Pritchard, C. R. McArdle, S. K. Wilson, The Stokes boundary layer for a power-law fluid, Journal of Non-Newtonian Fluid Mechanics 166 (2011) 745-753.

[21] G. K. Batchelor, An Introduction to Fluid Dynamics, Cambridge University Press, 1967.

[22] R. I. Tanner, Engineering Rheology, Oxford University Press, 2nd edition, 2000.

[23] P. Luchini, A. Bottaro, Linear stability and receptivity analyses of the Stokes layer produced by an impulsively started plate, Physics of Fluids 13 (2001) 1668-1678. 

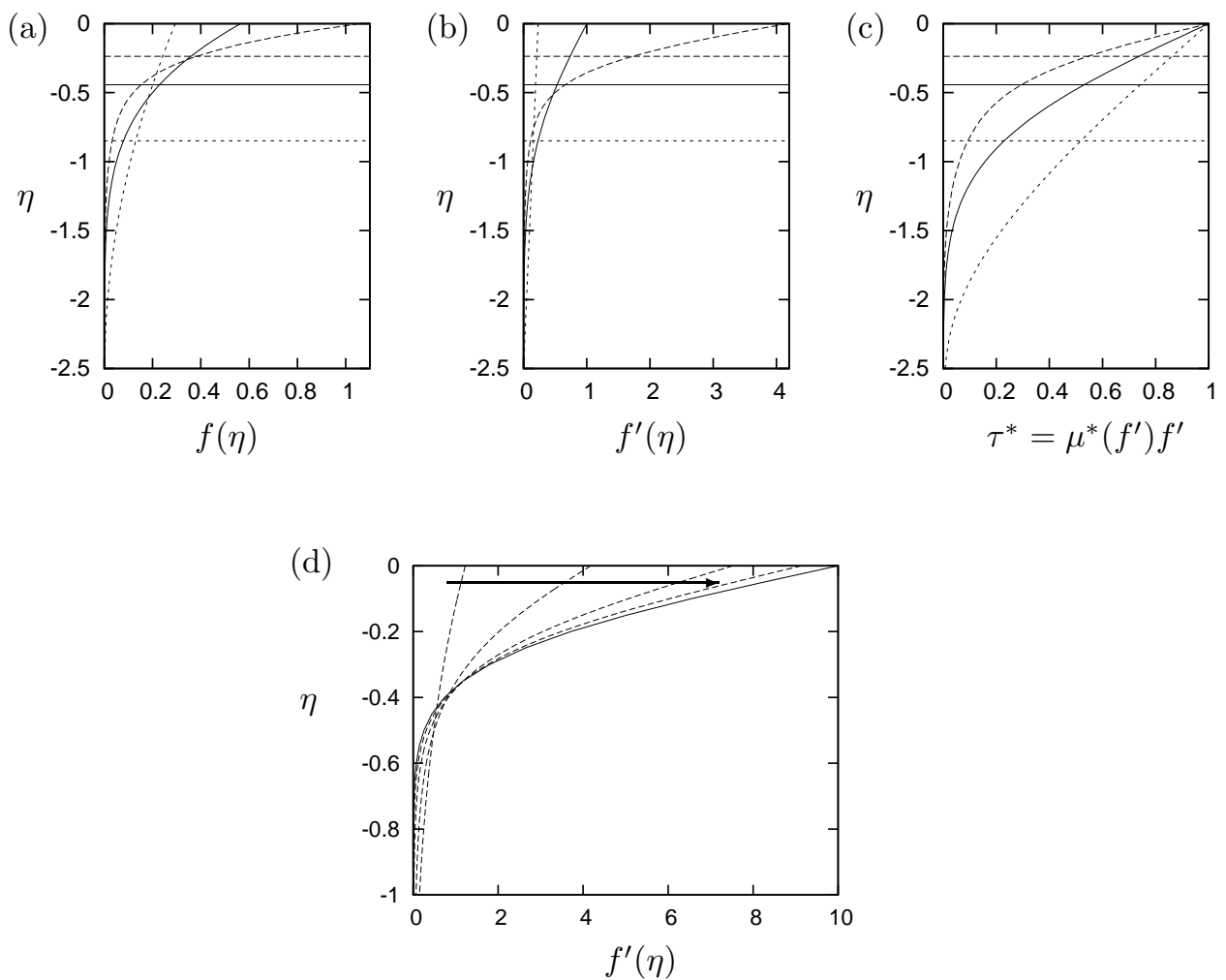

Figure 1: $(\mathrm{a}-\mathrm{c})$ Boundary layers for a Newtonian fluid (solid lines) and for Carreau fluids with $n=0.5$ and $\lambda^{*}=10$ together with $\mu_{\infty}^{*}=0.1$ (shear-thinning; heavy dashed lines) or $\mu_{\infty}^{*}=10$ (shear-thickening; light dashed lines): (a) velocity profile $f(\eta)$; (b) shear rate (vorticity) $q^{*}=f^{\prime}(\eta)=-\omega^{*} ;$ (c) shear stress $\tau^{*}=\mu^{*}\left(f^{\prime}(\eta)\right) f^{\prime}(\eta)$. The horizontal lines $\eta=-\eta_{\mathrm{d}}$ in each plot represent the displacement thickness for the Newtonian case $\left(\eta_{\mathrm{d}} \approx 0.443\right.$; solid lines), the shear-thinning Carreau fluid $\left(\eta_{\mathrm{d}} \approx 0.236\right.$; heavy dashed lines) and the shear-thickening Carreau fluid $\left(\eta_{\mathrm{d}} \approx 0.850\right.$; light dashed lines). (d) Near-wall behaviour of $f^{\prime}(\eta)$ for a Newtonian fluid with $\mu^{*}=0.1$ (solid line) compared with Carreau fluids (dashed lines) with $\mu_{\infty}^{*}=0.1, n=0.5$ and $\lambda=1,10,100$ and 1000; the arrow indicates the direction of increasing $\lambda$. 

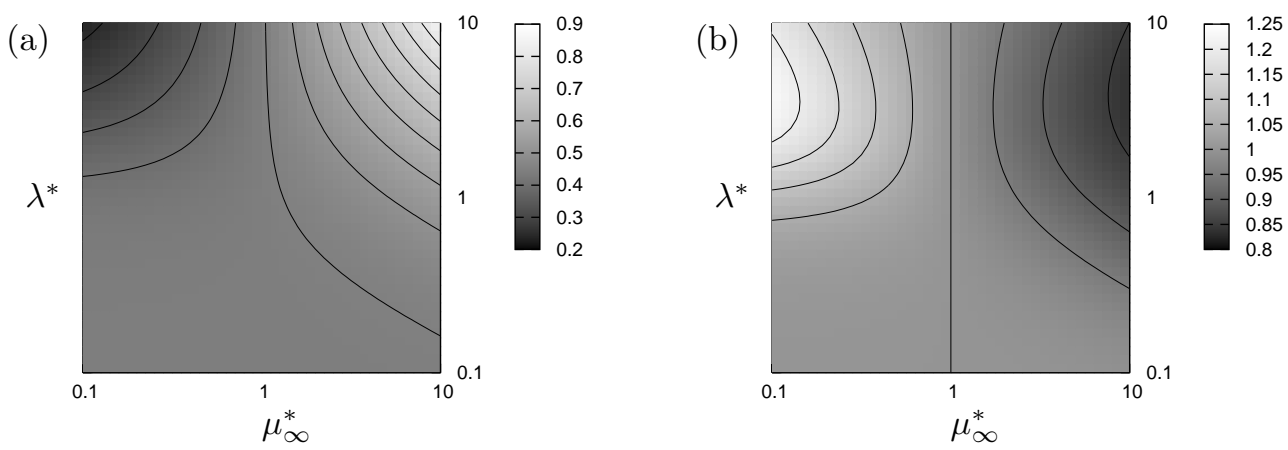

Figure 2: A Carreau fluid with $n=0.5$ : (a) the displacement-thickness coefficient $\eta_{\mathrm{d}}$, with contours at intervals of 0.05 from 0.25 to 0.8 ; (b) the nondimensionalisation-independent quantity $16 \mathcal{F} / \pi$, with contours at intervals of 0.05 from 0.85 to 1.2 . 
(a)

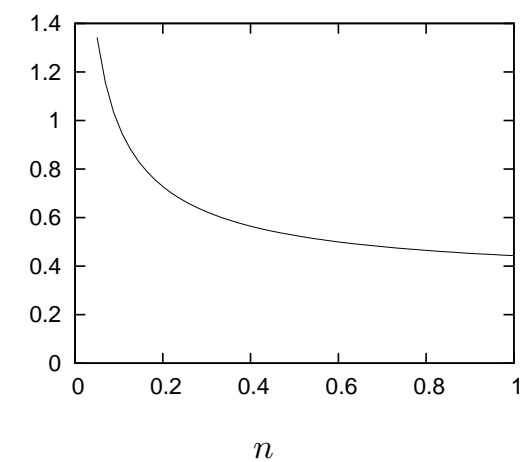

(b)

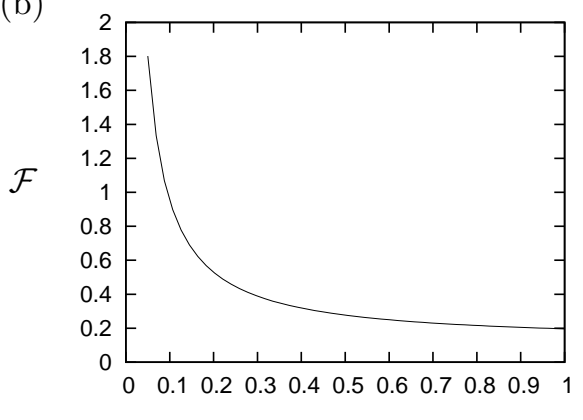

$n$

Figure 3: A shear-thinning power-law fluid: (a) the displacement-thickness coefficient $\eta_{\mathrm{d}}$; (b) the nondimensionalisation-independent quantity $\mathcal{F}$. 